\title{
Varieties of Analogical Reasoning
}

\author{
Robert R. Hoffman \\ Institute for Human and Machine Cognition \\ rhoffrman@ihmc.us
}

\author{
Thomas C. Eskridge \\ Institute for Human and Machine Cognition \\ teskridge@ihmc.us
}

\begin{abstract}
Motivation - The purpose of this article is to reinvigorate debate concerning the nature of analogy and broaden the scope of current conceptions of analogy. Research approach - An analysis of the history of the concept of analogy, case studies on the use of analogy in problemsolving, cognitive research on analogy comprehension, and a naturalistic inquiry into the various functions of analogy. Findings and Implications - Psychological theories and computational models have generally relied on: (a) A single set of ontological concepts (a property called "similarity" and a structuralist categorization of types of semantic relations) (b) A single form category (i.e., the classic four-term analogy), and (c) A single set of morphological distinctions (e.g., verbal versus pictorial analogies). The taxonomy presented here distinguishes functional kinds of analogy, each of which presents an opportunity for research on aspects of reasoning that have been largely unrecognized. Originality/Value - The various functional kinds of analogy will each require their own treatment in macrocognitive theories and computational models. Take away message - The naturalistic investigation of the functions of analogy suggests that analogy is a macrocognitive phenomenon derivative of number of supporting processes, including the apperception of resemblances and distinctions, metaphor, and the balancing of semantic flexibility and inference constraint.
\end{abstract}

\section{Keywords}

Analogy, cognitive research, problem solving, scientific reasoning, computer models

\section{INTRODUCTION}

As a strategy in decision making and sensemaking, as well as a rhetorical device, the analogy concept expanded greatly over the centuries since Aristotle discussed proportional analogies. In his System of Logic (1982, Ch.20), John Stuart Mill echoed Etienne de Condillac's definition of analogy, "the resemblance of relations." This definition is essentially the one that has been employed by modern philosophers, computer scientists, and cognitive scientists. Analogy, in one form or another, has widely been assumed to be a critical process in perception, language, learning, and problem solving (Hoyloak and Thagard, 1997; Schumacher and Gentner, 1988). Residing as it does "at the core of cognition" (Hofstadter, 2001). Analogy has been an important topic in the history, philosophy, and psychology of science, in cognitive science, and the field of artificial intelligence (see Holyoak and Barnden, 1994; Gentner, Holyoak and Kokinov, 2001, Komendzinski, 2001).

There is, to be sure, research showing that analogy is not ubiquitous and is not always helpful in problem solving, whether on the part of college students in psychology experiments or on the part of experts engaged in their domain tasks (Bearman, Ball and Ormerod, 2007; Donnelly and McDaniel, 2000; Klein and Calderwood, 1988; Kolodner, 2002; Spiro, Feltovich, Coulson and Anderson, 1989). This being said, analogy is indubitably a useful reasoning strategy. Indeed, it is possible to analyze the broad history of all sciences in terms of the major analogies (Hoffman, Cochran and Nead, 1990; Leatherdale, 1974).

Going even further, it is often claimed that analogy is a fundamental, basic, or irreducible mental operation or process (e.g., Rumelhart and Norman, 1981), and that analogy is just a form of similarity (e.g. Gentner and Markman, 2005; Holyoak, 2008a). In this article, we revisit traditional definitions of analogy, and modern approaches influenced by psychological research and computational modeling. Next, we deconstruct the traditional notion of analogy by comparing it to a number of functionally-distinct forms of analogical reasoning, each representing a phenomenon that can be empirically and computationally investigated in its own right. (A more extensive treatment appears in Hoffman, Eskridge and Shelly, in press.)

\section{ANALOGY IN PSYCHOLOGY AND COMPUTER SCIENCE}

Analogical reasoning has been the subject of numerous academic investigations by cognitive psychologists using a variety of materials (descriptions of hypothetical animals, patterns of colored squares, and so forth) and a variety of tasks (e.g., feature mapping, inference-making, sentence recall and recognition, and so forth) (e.g., Perrott, Gentner and Bodenhausen, 2005). Studies have been conducted on the conditions under which people 
will make analogical inferences during comprehension (e.g., Day and Gentner, 2007). Many studies have investigated the relative importance of relational versus featural similarity in the formation of analogical mappings (e.g., Gentner and Kurtz, 2006). Many studies have explored the conditions under which people will notice analogies and solve problems by analogical transfer, that is, whether people need hints, diagrams or other reasoning aids (e.g., Zamani and Richard, 2000). Transfer is most likely when surface or featural similarity is high and when there is only a brief delay between acquisition and test phases (cf. Wharton, et al., 1994).

Many attempts have been made to computationally model analogical reasoning, spanning many applicationsstory understanding, theorem proving, means-ends analysis in planning, the resolving of political disputes, etc. The many AI systems utilize a variety of paradigms including the deductive paradigm, the production rule paradigm, sequential versus parallel processing, Bayes nets, neural nets, and so on. Some models seem passive, that is, they generate all possible mappings or pairings of elements and then evaluate them for coherence and pare down the set of good candidates. But others seem more active or selective, say, by restricting mappings to those that satisfy given a priori goals. (For more details, see Hoffman and Eskridge, in press.)

Both computer science and much of the psychological research has focused on the A : B :: C: D form of analogy. Researchers present participants (typically, college students taking psychology courses) with preformulated problems that are structured, semantically limited, and usually context-free. This is the starting point for the Naturalist. The A : B :: C: D form is by no means the only form and function that analogy takes; different types of analogy occur in different contexts, and are used for different purposes.

\section{REASONING WITH THE GOAL OF CREATING ANALOGIES}

What we can call "Ad-hoc" (to this) analogy occurs in contexts in which the goal is to construct analogies de novo, typically in the explicit A : B :: C : D format, to meet certain criteria or to suit particular purposes. The purpose delimits the form of the analogies (e.g., geometrical, verbal, etc.), the categories or general domains for the terms, and the sorts of relations or transformations to be involved in the analogies. Analogies are created such that they have three terms, accompanied by a set of choice terms that might be completions such that only one of the alternatives is "correct" (i.e., it satisfies the intended relation). The analogies are then applied in their intended use, and their usefulness is somehow evaluated, according to measures or criteria that also stem from the initial purpose.

Ad-hoc analogies can be created so as to rely on essentially any form of relation-(Pharmacy : Drugs :: Grocery : Food), (Kitchen : Eat :: Bedroom : Sleep), (Deep : Shallow :: Expensive : Inexpensive), and so forth. The Miller Analogies Test is notorious for its mind-boggling cleverness. Having created a great many such analogies ourselves, it is apparent that Ad-hoc analogy requires mental effort and lots of trial-and-error, but there is far more to it in terms of motivation and affect. In running through this process of deliberately creating tricky analogies to meet certain criteria, one cycles through conjectures and criticisms, sometimes entirely abandoning one's path ("Maybe there are not enough letter sequences involving straight-straight-curved letters..."). Many people have engaged in this sort of reasoning, including all the people who make analogy items for intelligence tests and psychological experiments. But as far as we can tell, there is no research on how it is that people accomplish this - how they make analogies "out of the blue"—and there is limited theoretical extension to this in psychological theory and computer modeling.

\section{REASONING WITH THE GOAL OF SENSEMAKING}

Pre-hoc ("before this") analogies occur in discovery contexts, as in most scientific analogy. In Thagard's (1989) terms, the goal is to find coherent explanations, that is, to help one understand some given new concept or observation (and generate ideas for further hypothesis tests or observations). Gentner (1982) referred to a distinction between "explanatory" and "expressive" analogies. She argued further that explanatory analogies are specific, clear and systematic whereas expressive (or poetic) analogies are rich and idiosyncratic (p. 127). More recently, Lee and Holyoak (2008) demonstrated that the formation of causal mental models can drive the processes of realizing analogies and making inferences from them.

For Pre-hoc analogies, the goal and the "given" information differ from those in Ad-hoc analogy. For Pre-hoc analogy, all one really has at the start is the ill-defined topic concept, the thing to be explained or explanandum, as in questions like, "What are atoms?" or "What is heat?" A number of researchers have proposed am "encoding-retrieval-mapping" model (Holyoak and Barnden, 1994a,b; Holland, et al., 1986; Thagard, et al., 1990). The first step - retrieval — is, of course, where the miracle happens: How do you know to recognize an explananda that has explanatory potential unless you have already retrieved it from memory? 
Pre-hoc analogy involves missing data, ill-defined goals, and incomplete specification of parameters (Klein, 1987). A major gap is that AI models of analogy are typically not integrated with models of problem solving (Holyoak, 2008a). Models of analogy that construct mappings based on the matching of pre-ordained lists of semantic features have trouble with problem solving situations in which there are unknown or ill-defined factors. There might be some constraint-based mechanism for retrieving multiple candidate vehicles (Thagard, et al., 1990), but this just pushes the miracle a step forward. Models for Pre-hoc analogy also must include criteria for when to reject candidate analogies due to explanatory breakdown, some sort of a justification or evaluation processes (Falkenhainer, et al., 1990; Kedar-Cabelli, 1988; Winston; 1979), and some process of rerepresentation or "radical restructuring" (Chalmers, et al., 1992; Fass and Wilks, 1983; Gentner and Calhoun, 2008). Finally, computational models require some sort of learning mechanism, such as in systems that use Bayes nets and systems that hybridize symbolic and neural or connectionist architectures (e.g., Hoyloak, 2008b; Hummel and Holyoak, 2005).

\section{PUZZLE SOLVING OF INCOMPLETE ANALOGIES}

Post-hoc ("after this") analogies are ones that are given, perhaps already fleshed-out to some extent, and yet are waiting for explication or further analysis. One can attempt to discern "the" meaning of a Post-hoc analogy, defined in terms of the world knowledge of a human interpreter, or the semantics and inference processes within a computational model. The goal is to reveal the process of comprehending pre-formulated analogies or to suggest ways of forging computational models of the process of comprehending pre-formulated (as possibly incomplete) analogies, as in A : B :: C : ? Assumptions are made about the semantic base and the processes of inference constraint, and these can be expressed as a theory or implemented in a computational model. As far as we can tell, all psychological theories deal with the Post-hoc analogy situation, as for example in Sternberg's (1977a,b) ambitious program of reaction-time research.

\section{REASONING WITH THE GOAL OF UNDERSTANDING THE CONCEPT OF ANALOGY}

This form, which can be called Pro-hoc ("for this") analogy, is subtle. Pro-hoc analogies are ones that are selected intentionally for the purpose of assessing or refining a theory or model of Post-hoc analogy. Unlike Adhoc analogy, in which a creator thinks about the content of particular analogies, in the Pro-hoc context a theorist thinks about analogy as a concept, and about theories or models of it. This chapter is an exercise in Pro-hoc analogy. Pro-hoc analogy occurs whenever a Post-hoc analysis of given analogies has yielded a processing or computational model which is then tested-it should predict something and should do so better than some alternative model. Reasoning in the Pro-hoc context also occurs every time someone thinks about analogy as a concept.

We can illustrate by the Structure Mapping Engine (SME) (Falkenhainer, et al., 1990, Forbus, Ferguson, and Gentner, 1994). The SME stems from Gentner's (Gentner, et al., 1997) analyses of metaphor in science and from research on students' understanding of physical concepts. The SME system operates by mapping the relations that hold among the nodes within the base domain onto the relations that hold among the nodes within the target domain. Operations of the SME include retrieval of a domain for comparison based on similarity, mapping (of candidate inferences), and evaluation in terms of similarities - more similarities mean there's more "structure" in the mapping. The SME generates all possible relational mappings of source and target. Restriction comes in a final process in which an interpretation is chosen based on a numerical analysis that converges on maximal consistency. These two processes, directed search/selection and consistency evaluation, represent analogy in the Pro-hoc context.

\section{CONTRA-HOC ANALOGY: POKING HOLES IN ARGUMENTS AND THEORIES}

Contra-hoc ("against this") analogy is intended to show the flaws of an argument, including the rationale for a computational model. We find three distinct kinds of Contra-hoc analogy: Rebuttal analogy, Disanalogy, and Monster analogy.

\section{Rebuttal Analogy}

Rebuttal analogy is analogy used to persuade or rebut an argument, a commonly used rhetorical device. This is clearly distinct from explanatory analogy, since the goal is pragmatic, that is, to affect attitudes or behavior. Rebuttal analogy would presumably be of great interest in the fields of communication and rhetoric, but has not been much researched apart from the study of the rhetorical functions of metaphors (Whaley and Holloway, 1996). Brian Whaley surveyed some rebuttal analogies found in the news reports and determined that the main goal of a rebuttal analogy is to dispute someone else's claims. Rebuttal analogy can have a second purpose, to show by exaggeration that the opponent's argument is stupid and not just ridiculous. This is a ripe topic for research on the pragmatics and sociolinguistics of analogy. 


\section{Disanalogy}

A disanalogy is one that supports contradictory or mutually exclusive conclusions. Disanalogy goes beyond the idea that analogies are always incomplete or limited, to the idea that a single analogy can generate contradictory arguments. "In general, cognitive models of analogy have been built on the assumption that incompatible but causally supported inferences do not follow from a single analogy" (Shelly, 2002, p. 82).

Cameron Shelly $(2002,2003)$ performed a formal analysis (using the representational schemes of Holyoak and Thagard, 1997; and Gentner, 1983) of a number of scientific and philosophical dialogs. Shelly showed that disanalogy involves adding into an analogy one or more additional relational predicates, resulting in a conclusion that is contradictory to that conclusion made from the original analogy.

Here is an example we adapt from a recent discussion among some political pundits.

Commentator 1: The American economy has been a strong producer for two hundred years. The last thing you want to do in this economy is kill the goose that laid the golden eggs.

In context, the implication was that the US should not create more regulations or "socialize" the banking system.

Commentator 2: Yes, but the goose is sick and so you have to intervene somehow.

In context, the implication was that some additional controls are needed. Since the disanalog-based rebuttal is a variation on the source analogy, the proponent of the original analogy is closed off from objecting.

In looking at these kinds of Contra-hoc analogies and comparing them to those that inhabit the literatures of cognition and computational science, Shelly (2002) and Veale and Keane (1997) suggested that cognitive and computational models of analogy have been biased by the focus on a certain form of analogy and selection of certain kinds of cases. This point is placed in strong relief by Monster analogies.

\section{Monster Analogy}

Analogy can involve multiple formats and a boundless variety of semantic and conceptual relations (Hoffman, 1995). Monster analogies were intended to serve as grounds for disputation of the assumptions and postulates underlying cognitive and computational models of analogy. This functional type of analogy puts us right up against the problems of creativity, "syntactic infinity" (Katz and Fodor, 1963) and "semantic infinity" (Hoffman and Honeck, 1976). Table 1 presents some examples of monster analogies.

\section{Table 1. Example monster analogies.}

\begin{tabular}{|l|l|}
\hline \multicolumn{1}{|c|}{ Analogy } & \multicolumn{1}{c|}{ Explanation } \\
\hline $\begin{array}{l}\text { Plato's analogies: Philosophy :: Maxwell's analogies : } \\
\text { Physics }\end{array}$ & Analogy can be about analogy. \\
\hline River : Story :: Milk: ? & $\begin{array}{l}\text { Such anomalous analogies can be solved, if people are } \\
\text { given latitude (Johnson, 1975). }\end{array}$ \\
$\begin{array}{l}\text { Horse : Time :: Stone : } \\
\text { (a) king, (b) book, (c) girl, (d) train }\end{array}$ & $\begin{array}{l}\text { Analogies can be self-referential and ill-formed. } \\
\text { Because the analogy is correct in asserting its own ill- } \\
\text { formedness, does it really have an anomalous } \\
\text { semantics? analogy : Ill-formed syntax :: Ill-formed } \\
\text { semantics : Horse. }\end{array}$ \\
$\begin{array}{l}\text { "This analogy refers to itself" : Self-reference :: "This } \\
\text { analogy does not refer to itself" : Contradiction }\end{array}$ & \begin{tabular}{l} 
This second example presents a similar conundrum. \\
\hline $\begin{array}{l}\text { (Feathers : Birds :: Hair : Mammals) : Simple :: This } \\
\text { analogy: Complex }\end{array}$
\end{tabular} \\
\hline
\end{tabular}

\section{ANALOGIZING AS A CONTINUOUS PROCESS}

Cognitive theories of analogical reasoning are dynamic insofar as they posit hypotheses about mental processes that are placed into a causal sequence. The notion of Trans-hoc ("across this") analogy is that there is not always a clear-cut point in time where one can definitively say that a process called analogical reasoning has been engaged. Nor is there always a clear-cut ending to analogical reasoning where one can say that an analogy has been "solved." Must cognitive theories terminate when the analogy is understood or a mapping is deemed coherent? Perhaps the drive toward termination is a consequence of the sorts of things that psychological theories like to measure, such as reaction time. 
Trans-hoc analogy is actually a common form of analogy, perhaps the most common. And yet, it requires a rather different sort of theory from all the other forms of analogical reasoning. In addition, any of the other goalrelated forms (Ad-hoc, Pro-hoc, Contra-hoc, etc.) can be manifested in the Trans-hoc process. In Trans-hoc analogy, conceptualization-conjecture is repeated. Unresolved "mappings" remain unresolved. Reasoning may be interrupted for any of a variety of reasons and for any length of time. Candidate understandings or concepts can be tentatively adopted, that is, put to some use. Those understandings or concepts can subsequently undergo even further refinement even though they have been put to some use.

\section{IMPLICATIONS}

We have discussed some functionally distinct kinds of analogizing. These mandate differing treatments in psycholinguistics, sociolinguistics, and computational modeling, since they involve:

- Differing goals,

- Differing kinds of "given" information,

- Differing kinds of constraint,

- Differing styles of justification and criticism, and

- Differing purposes, spanning pragmatics or rhetoric, and problem solving.

For computational modeling, each involves differing kinds of sequences of operations - if one chooses to think in terms of microcognition and stage theories—or differing kinds of or cycles and parallelisms, if one prefers to take a macrocognitive perspective.

This naturalistic investigation highlights the fact that only certain aspects of analogical reasoning have come under scrutiny in psychological research, and have been modeled computationally. In general, AI models and cognitive theories have as their goal the Post-hoc analysis (i.e., "build me a theory") of incomplete Ad-hoc or Pre-hoc (i.e., pre-formulated) verbal analogies so as to generate "solutions"—-single candidate completions (literal paraphrases) that satisfy certain Pre-hoc (explanatory) and Pro-hoc (i.e., justification) criteria, and thereby terminate the analysis.

Furthermore, analogy has been reified. Given that analogy (especially the four-term analogy used in mental tests) has become ingrained in Western civilization, it is now possible to claim that analogy is necessary for problem solving precisely because the concept of analogy and the analogy format were invented to label and describe exactly the sorts of phenomena that problem solving involves (see Sacksteder, 1979, p.35; and Shelly; 2002, p. 95). Many bold claims serve as the bedrock of the literature (e.g., Bowdle and Gentner, 2005; Holyoak, 2008; Markman and Gentner, 2000). For example:

- Both metaphor and proverbs car be sufficiently understood as being mappings based on similarity.

- Analogy is a single and fundamental mental operation, even innate and universal,

- A process called analogical transfer underlies problem solving in general,

- Similarity itself is a fundamental cognitive process.

- $\quad$ Linguistic forms "have" meanings or semantic features, and that when regarded pair wise they possess a property called "similarity."

The mental operations foundational to analogical thinking seem to include a number of mysteries of macrocognition:

(1). The apperception of resemblances and distinctions,

(2). The human ability to create well-defined or analytical formats for laying out propositions that express meanings and perceptions,

(3). Mental imagery,

(4). Metaphor,

(5). Semantic flexibility, and

(6). Inference constraint.

Analogy does not exist in the sense that it is clearly and consistently distinguishable from these macrocognitive phenomena, except when it is a derivative of this set cast in stone as the classic four-term analogy. Broadening the scope of research, theory, and modeling may be helpful as cognitive science and AI grapple with the fundamental mysteries of macrocognition-mysteries that not only seem to be the underpinnings of so-called analogical reasoning, but that are also linchpins in strong definitions of artificial intelligence.

\section{REFERENCES}

Bearman, C. R., Ball, L. J. and Ormerod, T. C. (2007). The structure and function of spontaneous analogizing in domain-based problem solving Thinking and Reasoning, 13, 273-294.

Day, S. B. and Gentner, D. (2007) Nonintentional analogical inference in text comprehension. Memory \& Cognition, 35, 39-49. 
Donnelly, C. M., and McDaniel, M. A. (1993), "Use of analogy in learning scientific concepts," Journal of Experimental Psychology: Learning, Memory and Cognition, 19, 975-987.

Falkenhainer, B., Forbus, K.D., and Gentner, D. (1990). The structure-mapping engine: Algorithm and examples. Artificial Intelligence, 41, 1-63.

Gentner, D. (1982). Are scientific analogies metaphors? In D. S. Miall (Ed.), Metaphor: Problems and perspectives (pp. 106-132). Atlantic Highlands, NJ: Humanities Press.

Gentner, D. and Colhoun, J. (2008). Analogical processes in human thinking and learning. In A. von Müller \& E. Pöppel (Series Eds.) and B. Glatzeder, V. Goel and A. von Müller (Vol. Eds.), On thinking: Vol. 2. Towards a theory of thinking. Springer-Verlag Berlin Heidelberg.

Gentner, D., Holyoak, K. J. and Kokinov, B. N. (Eds.) (2001). The analogical mind: Perspectives from cognitive science. Cambridge, MA: Bradford Books.

Gentner, D. and Kurtz, K. J. (2006. Relations, objects and the composition of analogies. Cognitive Science, 30 , 609- 642 .

Hoffman, R. R., Cochran, E. L., and Nead, J. M. (1990). Cognitive metaphors in experimental psychology. In D. Leary (Ed.), Metaphors in the history of psychology (pp. 173-227). Cambridge: Cambridge University Press.

Hoffman, R. R., Eskridge, T. C and Shellly, C. (2009). A naturalistic investigation of some of the forms and functions of analogizing. Metaphor and Symbol.

Hofstadter, D. R. (2001). Analogy as the core of cognition. In D. Gentner, K. J. Holyoak, and B. N. Kokinov (Eds.), The Analogical mind: Perspectives from cognitive science (pp. 499-538).Cambridge, MA: Bradford Books.

Holland, J. H., Holyoak, K. J., Nisbett, R. E. and Thagard, P. R. (1986). Induction: Processes of inference, learning, and discovery. Cambridge: MIT Press.

Holyoak, K. J. (2008a). Analogy. Chapter 6 in K. J. Holyoak and R. G. Morrison (eds.), Cambridge handbook of thinking and reasoning (pp. 117-142). New York: Cambridge University Press.

Holyoak, K. J. (2008b). Induction as model selection. Proceedings of the National Academy of Science USA, 105, pp. 10637-10638.

Holyoak, K. J. and Barnden, J. A. (Eds.) (1994a). Advances in connectionist and neural computation theory, Volume 2: Analogical connections. Norwood, NJ: Ablex.

Holyoak, K.J. and Barnden, J. A. (1994b). Introduction. In K. J. Holyoak and J. A. Barnden, J. A. (eds.), Advances in connectionist and neural computation theory, Volume 2: Analogical connections (pp. 1-27). Norwood, NJ: Ablex.

Holyoak, K. J. and Barnden, J. A. (Eds.) (1994). Advances in connectionist and neural computation theory, Volume 2: Analogical connections. Norwood, NJ: Ablex.

Holyoak, K. J. and Thagard, P. (1997). The analogical mind. American Psychologist, 52, 35-44.

Hummel, J. E., \& Holyoak, K. J. (2005). Relational reasoning in a neurally-plausible cognitive architecture: An overview of the LISA project. Current Directions in Cognitive Science, 14, 153-157.

Klein, G. A. (1987). Applications of analogical reasoning. Metaphor and Symbolic Activity, 2, 201-218.

Klein, G. and Calderwood, R. (1988). How do people use analogues to make decisions? In Proceedings of the 1988 Case-based Reasoning Workshop_(pp. 209-223). Washington, DC: Defense Advanced Research Projects Agency.

Kolodner, J. L. (1983). Towards an understanding of the role of experience in the evolution from novice to expert. International Journal of Man-Machine Studies, 19, 497-518.

Komendzinski, T. (Ed.) (2001). Special issue on analogy. Theoria et Historia Scientarium.

Leatherdale, W. H. (1974). The role of analogy, model, and metaphor in science. Amsterdam: North-Holland.

Lee, H. S. and Holyoak, K. J. (2008). the role of causal models in analogical inference. Journal of Experimental Psychology, Learning, Memory, and Cognition, 34, 1111-1122.

Mill, J. S. (1878). System of Logic. London: Longmans, Green and Co.

Perrott, D. A., Gentner, D. and Bodenhausen, G. V. (2005). Resistance is futile: the unwitting insertion of analogical inferences in memory. Psychonomic Bulletin and Review, 12, 696-702.

Rumelhart, D. E. and Norman, D. A. (1981). Analogical processes in learning. In J. R. Anderson (Ed.), Cognitive skills and their acquisition (p.. 335-360). Hillsdale, NJ: Erlbaum. 
Shelly, C. (2003). Multiple analogies in philosophy and science. Amsterdam: John Benjamins.

Schumacher, R. M. and Gentner, D. (1988). Transfer of training as analogical mapping. IEEE Transactions on Systems, Man, and Cybernetics, 18, 592-600.

Spiro, R. J., Feltovich, P. J., Coulson, R. L. and Anderson, D. K. (1989). Multiple analogies for complex concepts: Antidotes for analogy-induced misconception in advanced knowledge acquisition. In S. Vosniadou and A. Ortony (Eds.), Similarity and analogical reasoning (pp.498-531). New York: Cambridge University Press.

Thagard, P. (1989). Explanatory coherence. Behavioral and Brain Sciences, 12, 435-467.

Thagard, P., Holyoak, K. J., Nelson, G. and Gochfeld, D. (1990). Analog retrieval by constraint satisfaction. Artificial Intelligence, 46, 259-310.

Wharton, C. M., Holyoak, K. J., Downing, P. E., Lange, T. E., Wickens, T. D. and Melz, E. R. (1994). Below the surface: Analogical similarity and retrieval competition reminding. Cognitive Psychology, 26, 64-101.

Zamani, M. and Richard, J.-F. (2000). Object encoding, goal similarity, and analogical transfer. Memory and Cognition, 28, 873-886. 